\title{
Multidimensional Image Compression through Discrete Wavelet Transform in Matlab using Daubechies Wavelet
}

\author{
Nazir Jan \\ Department of Computer Systems \\ Engineering, University of \\ Engineering and Technology \\ Peshawar, Pakistan
}

\author{
Nasruminallah \\ Department of Computer Systems \\ Engineering, University of \\ Engineering and Technology \\ Peshawar, Pakistan
}

\author{
Rehmatullah \\ Department of Computer Systems \\ Engineering, University of \\ Engineering and Technology \\ Peshawar, Pakistan
}

\begin{abstract}
This research paper unveils the most powerful and latest tool for image compression; called Wavelet Transform (WT).WT avoids the blocking artifact of conventional DCT Transform. Fourier Transform is the famous transform but this transform always lost time information and preserves only frequency information. Wavelet Transform reserves the information of both time and frequency domains. WT is based on Function approximation or mathematical polynomials instead of blocks like DCT. Matlab simulations, in this research paper, shows much satisfying results and excellent compression ratio when applied on a multidimensional image using multiband Wavelet transform. Daubechies wavelet has been selected in the proposed research paper as analyzing signal.
\end{abstract}

\section{General Terms}

Image Processing

\section{Keywords}

Daubechies Wavelet, Multiband multidimensional image compression, multimedia data, Wavelet Transform

\section{INTRODUCTION}

Last decade or so has been the era of information explosion with the need of huge data and multimedia storage and like audio, video, images and text would have been thought as an "impossible work" to store and transmit without using compression techniques which can achieve even 95\%compression ratio. There have been quite a list of compression techniques developed during last few decades. Examples are DCT, JPEG, MPEG etcetera where some are lossless and some are losing compression techniques. These techniques use different tactics to encode multimedia data and then compress that data. Some deficiencies have been found out in all techniques developed so far. For example we have blocking artifact in DCT and non-satisfactory compression ratio in JPEG and many more like these. Wavelet Transformation proved to be the latest, error free and most powerful image and video compression methodology. The wavelet transform is basically the further refinement of another transform called sub band transform and is becoming very popular for image and video coding. The wavelet transform is based on the approximation theory.

\section{WAVELET TRANSFORM THEORY}

Wavelet transform is based on the approximation theory. This means wavelet transform, unlike blocked based transforms, represents an image in the form of mathematical functions or polynomials. Each function is then represented and transmitted separately. These functions are extracted or derived from signal spectrum (the signal spectrum basically represents an image) with the help of filter banks like Low pass filter or high pass filter. The following subsections explains in detail how an image is represented as mathematical function and how it is passed through bank of filters. Mathematically;

$$
X_{w(a, b)}=\int_{-\infty}^{\infty} x(t) \Psi_{a, b}(t) d t
$$

The time Dilated and translated version of Bases Function is as follow;

$$
\Psi_{\mathrm{a}, \mathrm{b}}=\frac{1}{\sqrt{\mathrm{a}}} \Psi\left(\frac{\mathrm{t}-\mathrm{b}}{\mathrm{a}}\right)
$$

The basic function width is variable with respect to time dilate factor represented in the above formula as "a", also called scale. If we keep widening scale factor "a", the basic function gets wider and wider in the time domain but narrower and narrower at the same time in frequency domain and that's the property which makes wavelet superior to all its counter parts.ie wavelet works on both time and frequency domains at the same time. This property of wavelet transform makes it ideal for analyzing natural images and signals features of different sizes.

Example: a person looking through a window of a certain house will be best analyzed by the basis function with quite large "a", the window with a small "a" and the eyes of the person with even smaller "a" thus working exactly like frequency domain analysis with varying central frequency.

\section{REPRESENTATION OF A SIGNALTHROUGH DAUBECHIES WAVELET}

The scaling function of daubechies mother wavelet is given as below;

$$
f(t)=\left\{\begin{array}{cl}
-1 & 0 \leq t \leq 1 \\
0 & \text { otherwise }
\end{array}\right.
$$

And the coefficients are;

$$
C_{n}=\left\{\begin{array}{lr}
\frac{1}{\sqrt{2}} & n=0,1 \\
0 & \text { otherwise }
\end{array}\right.
$$

Thus daubechies function is obtained as below;

$\Psi_{(t)}=\phi(2 \mathrm{t})-\phi(2 \mathrm{t}-1)$ where $\Psi_{(t)}$ shows daubechies function and $\phi(\mathrm{t})$ represents its corresponding scaling function 

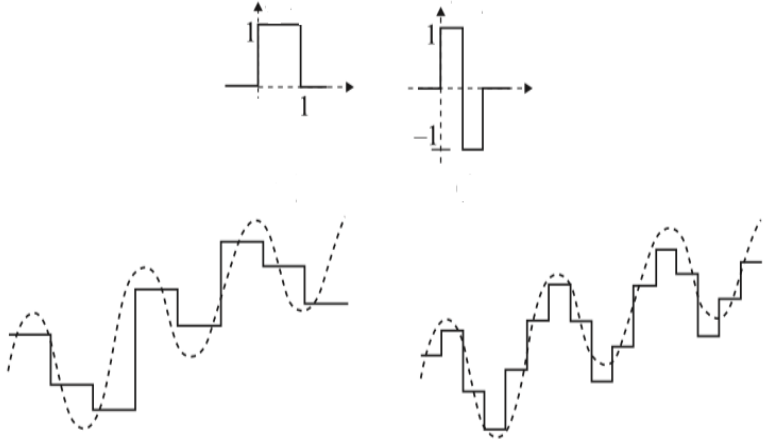

Figure 1 Daubechies function

So a natural image can be converted to a continuous time signal using function approximation through daubechies mother wavelet in the above figure 1

\section{EXPLANATION THROUGH BLOCK DIAGRAM}

Multiband, multidimensional Wavelet transform can be best explained with the given figure. Image is provided at the input gate first. Since it's a multidimensional image, High pass filter $\mathrm{H}$ and Low pass Filter $\mathrm{F}$ are applied on rows at first stage. The output of high pass filter gives an image with preserved high frequencies also called horizontal details image. The output from low pass filter gives an image with average details also called approximation details image. In the second stage the same filters are applied on the two sub images' columns which gives four sub images HH1, HL1, LH1 and LL1.HH1 sub image keeps Diagonal details, HL1 keeps horizontal details, LH1 keeps vertical details and LL1 keeps average details of the input image.LL1 is the most information carrying image, also called approximation image, which preserves all the low frequencies details, as most of the information are laid down in low frequencies in a natural image. The other three sub images can be put to zero for compression and efficient transmission. For further compression, the approximation image LL1 is fed again in the filter bank and the same process is repeated again which gives further sub images HH2, HL2, LH2 and LL2 .LL2 is our next approximation image and can be further compressed.

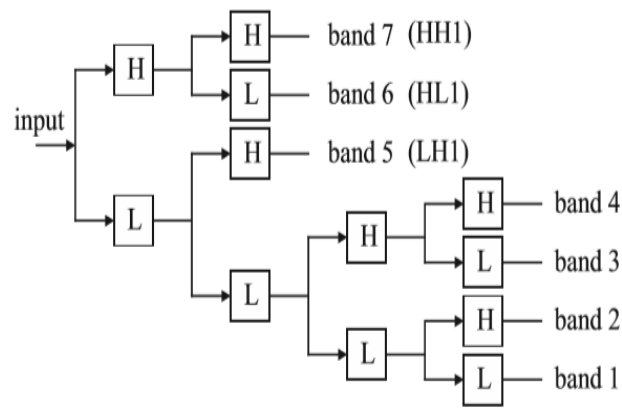

Figure 2 Block diagram

\section{SIMULATIONS AND RSULTS}

Wavelet transform function "dwt2"is applied on input images. The natural image is first represented in the form of a mathematical function like Haar, Morlet or Daubechies function. Now instead of blocks we have Functions to process. Function is then passed through filter bank to get the important and less important details separated. The less important details are set to zero and the important ones are preserved and further processed. In our discussion LL1 and LL2 are the most important details while all other sub images are non-important details and set to zero. Thus, compression is achieved. The figures and digits are given below with information in the table 1 .

Table 1 Experimental Results

\begin{tabular}{|c|c|c|}
\hline S.No & Ratio name & Compression ratio \\
\hline 1. & $\mathrm{Cr} 1$ & 0.3417 \\
\hline 2. & $\mathrm{Cr} 2$ & 0.0982 \\
\hline 3. & $\mathrm{Cr} 3$ & 0.0012 \\
\hline
\end{tabular}

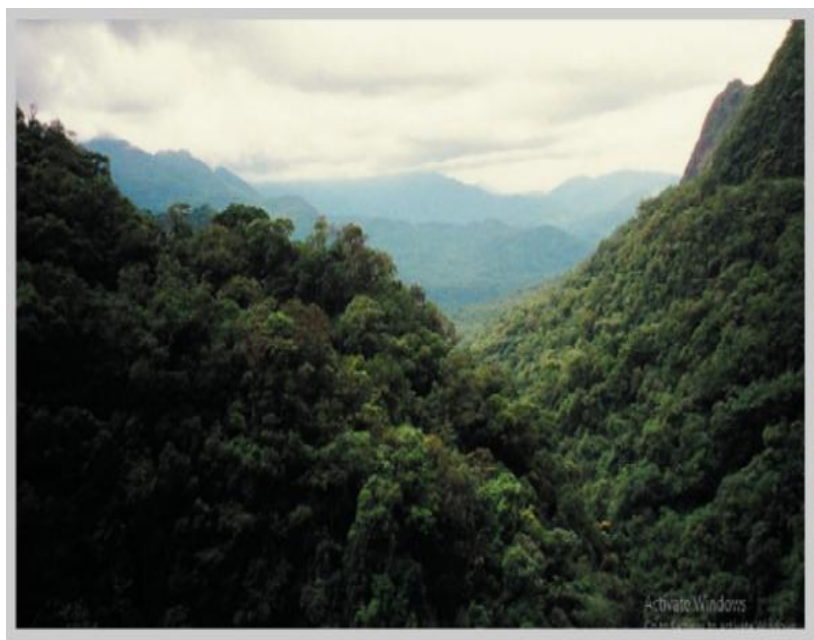

Figure 3 Input image

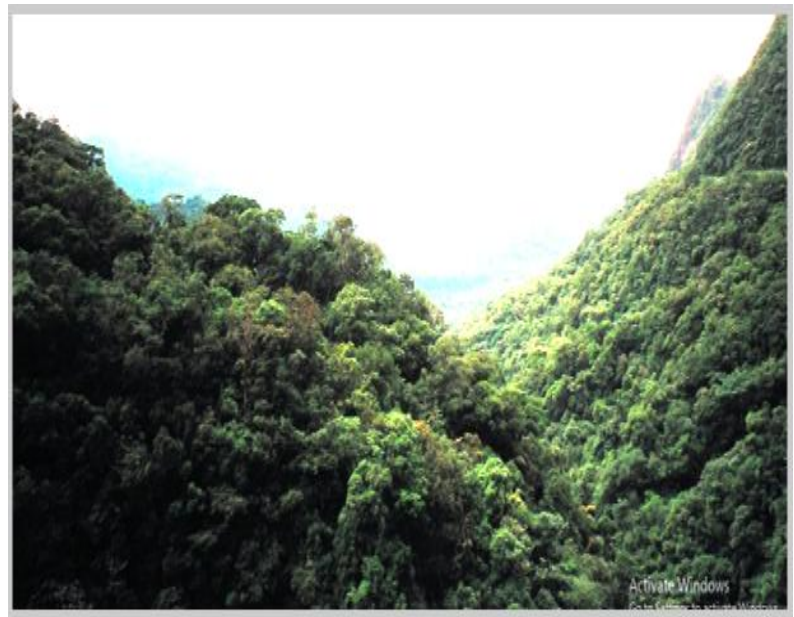

Figure 4 approximation image 


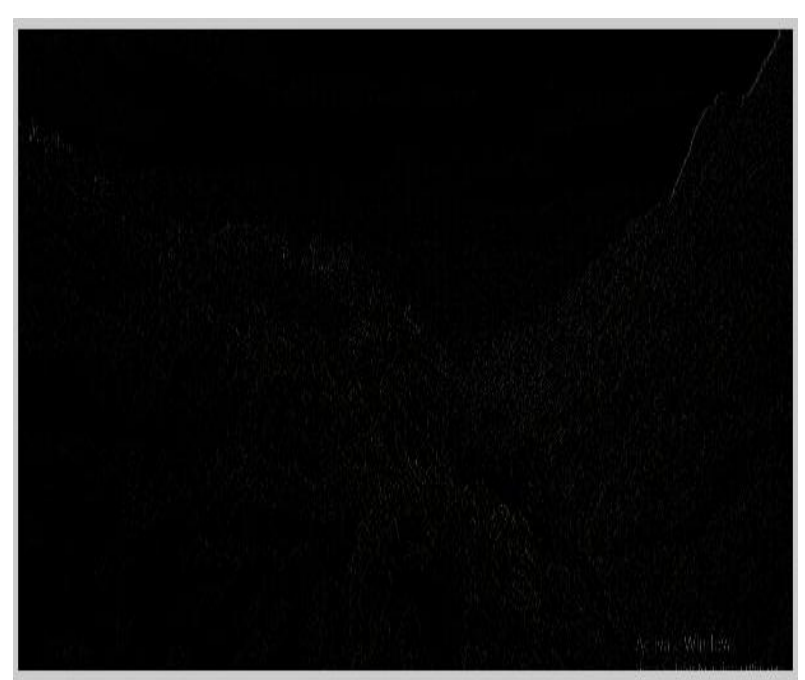

Figure 5 Diagonal image

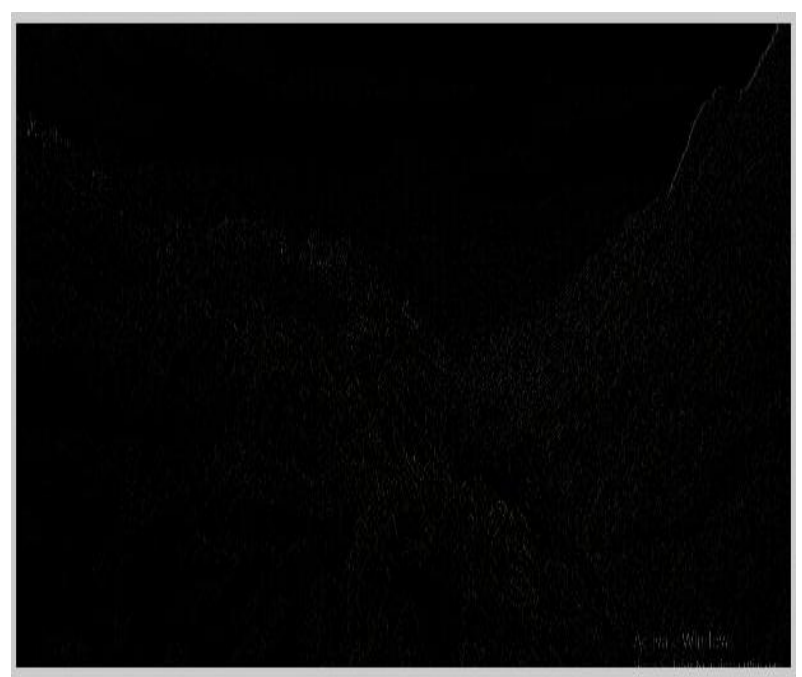

Figure 6 Vertical image

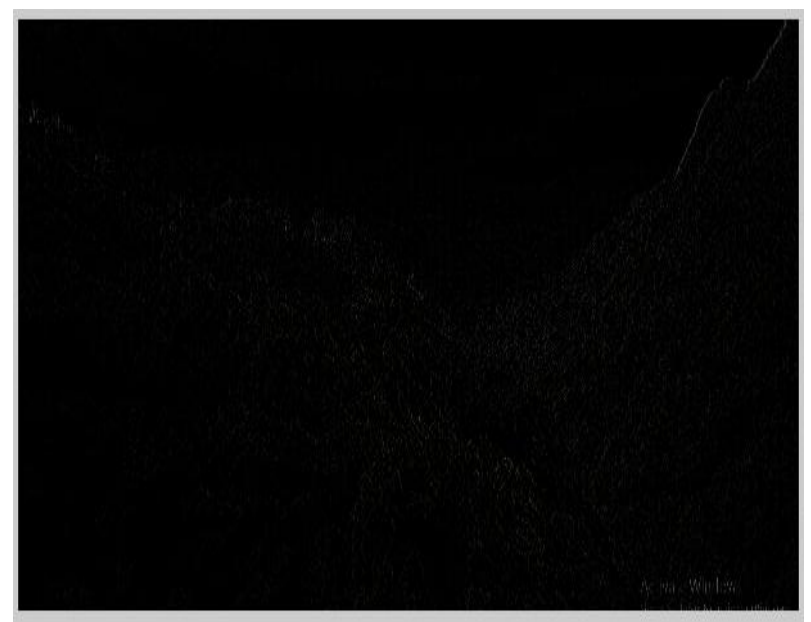

Figure 7 Horizontal image

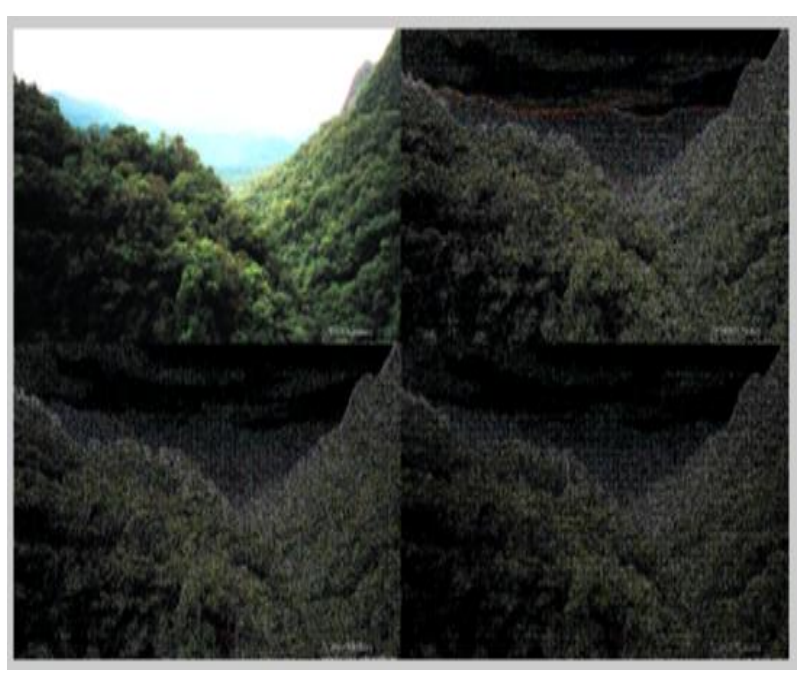

Figure 8 Combined image

\section{SECOND LEVEL DECOMPOSITION}

In the next iteration, Three more detailed images and one more approximate image are generated as shown in the following figures

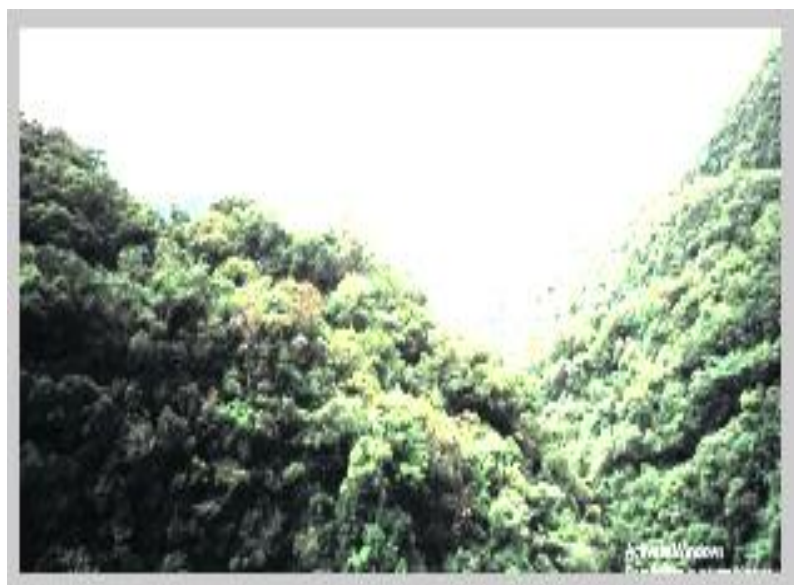

Figure 9 Approximation image of second level

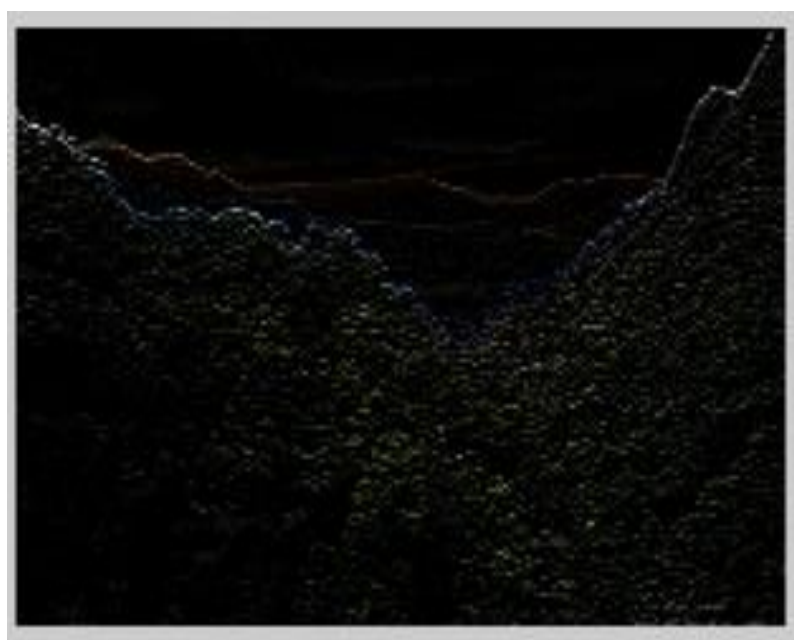

Figure 10 Diagonal image of second level 


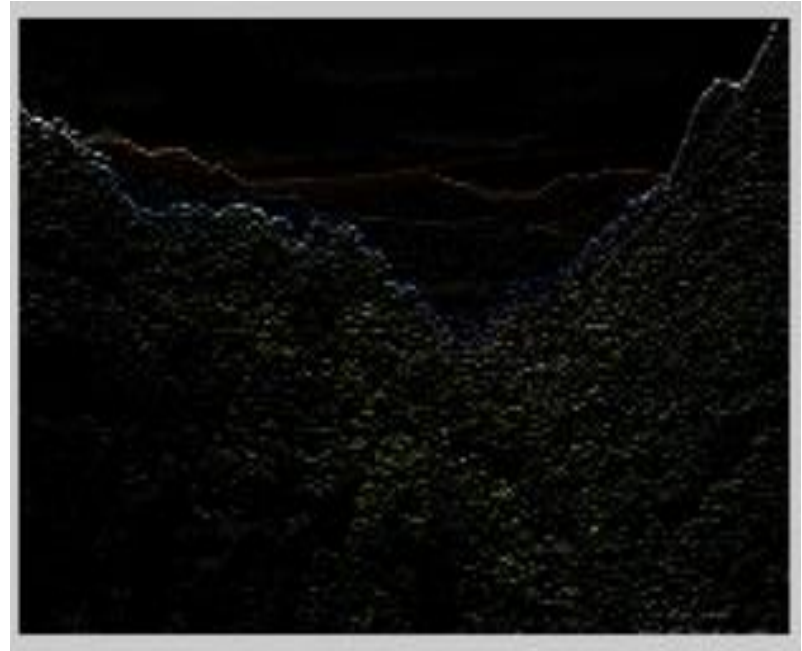

Figure 11 Vertical image of second level

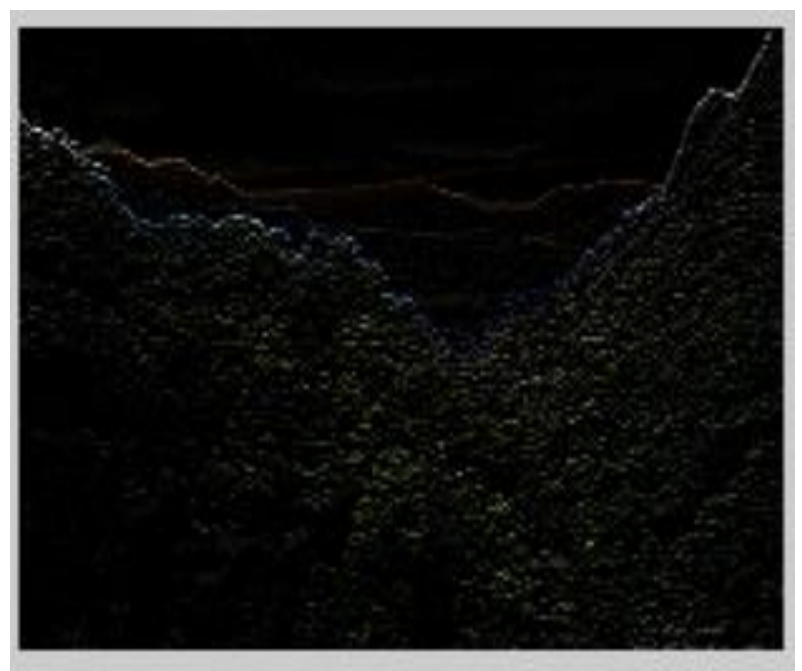

Figure 12 Horizontal image of second level

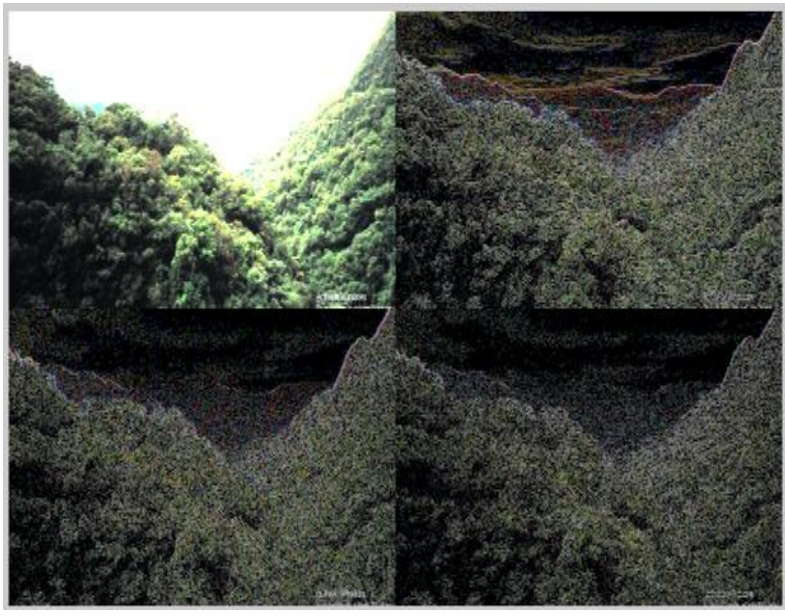

Figure 13 Combined image of second level

\section{REFERENCES}

[1] Gonzalez, R. C. and Woods, E. R. January 2002. Digital Image Processing (2nd Edition). Prentice Hall.

[2] Mallat, S. G. July 1989. A theory for multiresolution signal decomposition: the wavelet representation. vol. 11, pp. 674-693,

[3] Shapiro, J. M. Dec 1993. Embedded image coding using zerotrees of wavelet coefficients. vol. 41, pp. 3445-3462,

[4] Zhang, Y. S. 2008. Multiresolution analysis for image by generalized 2-d wavelets. Master's thesis,

[5] Daubechies, I. 1992. Ten lectures on wavelets, CBMSNSF Regional Conference Series in Applied Mathematics. Philadelphia, PA: Society for Industrial and Applied Mathematics (SIAM). vol. 61.

[6] Taubman, D. Oct. 24-28, 1999. High performance scalable image compression with ebcot. International Conference on Image Processing ICIP 99, vol. 3, pp. 344-348,

[7] Mallat, S. December 2008. A Wavelet Tour of Signal Processing, 3rd ed., Third Edition: The Sparse Way. Academic Press, 3 ed. 